\title{
Baixo peso ao nascer em duas coortes de base populacional no Sul do Brasil
}

\author{
Low birthweight in two population-based cohorts \\ in southern Brazil
}

Bernardo L. Horta 1

Fernando C. Barros 1

Ricardo Halpern 1

Cesar G. Victora 1

\footnotetext{
1 Departamento de Medicina Social e Materno-Infantil, Universidade Federal de Pelotas. C. P. 464, Pelotas, RS, 96001-970, Brasil.
}

\begin{abstract}
Low birthweight infants (under 2500 g) are much more likely to have severe diseases or die in the first year of life. This study's objective was to compare occurrence of low birthweight, preterm births, and intrauterine growth retardation in two cohorts born in the years 1982 and 1993 in Pelotas, Southern Brazil. All hospital births (corresponding to over 99\% of total births) were evaluated for both years. Low birthweight increased from $9.0 \%$ in 1982 to $9.8 \%$ in 1993 ( $p=0.2)$, preterm births increased from $5.6 \%$ to $7.5 \%$ ( $p<0.01)$, and intrauterine growth retardation increased from 15.0\% in 1982 to $17.5 \%$ in 1993 ( $p<0.05$ ). In the two years under study, family income was inversely associated with low birthweight and intrauterine growth retardation, but not with preterm births. Compared to 1982, mothers in the 1993 birth cohort had better socioeconomic and nutritional status, as well as better coverage of prenatal care. However, these improvements were not expressed as a decrease in low birthweight. On the contrary, after the possible confounding effects of socioeconomic and nutritional variables were controlled, risk of low birthweight in 1993 was 33\% higher than that of $1982(p<0.01)$.
\end{abstract}

Key words Low Birthweight; Child Health; Epidemiology; Growth; Nutrition

Resumo As crianças com baixo peso ao nascer (menos de $2.500 \mathrm{~g}$ ) apresentam um risco muitas vezes maior de morrer ou adoecer no primeiro ano de vida. O presente estudo teve como objetivo comparar a ocorrência de baixo peso ao nascer, nascimento pré-termo e retardo de crescimento intra-uterino, nos anos de 1982 e 1993, em Pelotas, Rio Grande do Sul. Nestes dois anos foram avaliados todos os nascimentos hospitalares, que representam mais de 99\% do total dos nascimentos. O baixo peso ao nascer aumentou de 9,0\% para 9,8\% em 1993 ( $p=0,2)$, os nascimentos pré-termo aumentaram de 5,6\% em 1982 para 7,5\% em 1993 ( $p<0,01)$ e o retardo de crescimento intra-uterino passou de 15,0\% em 1982 para 17,5\% em 1993 ( $p<0,05)$. Nos dois períodos estudados, a renda familiar esteve inversamente associada com o risco de baixo peso e retardo de crescimento intra-uterino, mas não com nascimentos pré-termo. Em 1993, apesar da melhoria nas condições sócio-econômicas e nutricionais das mães, como também do aumento no número de consultas pré-natais, observou-se um crescimento na prevalência de baixo peso ao nascer que não foi significativa na análise bivariada. Entretanto, após ajuste para possíveis variáveis de confusão, por meio de regressão logística, houve um aumento no risco de baixo peso ao nascer da ordem de 33\% $(p<0,01)$.

Palavras-chave Baixo Peso ao Nascer; Saúde da Criança; Epidemiologia; Crescimento; Nutrição 


\section{Introdução}

O peso de nascimento é o fator isolado mais importante na determinação da sobrevivência infantil, pois crianças com baixo peso (menos de $2.500 \mathrm{~g}$ ) apresentam um risco muitas vezes maior de morrer ou adoecer no primeiro ano de vida (McCormick, 1985). Por isso, o baixo peso ao nascer tem sido alvo de vários estudos epidemiológicos com o objetivo de identificar os seus fatores de risco, na tentativa de elaborar intervenções que possam reduzir estes fatores e prevenir a ocorrência do mesmo.

A importância do baixo peso ao nascer para a saúde pública é determinada não apenas pelos riscos subseqüentes de mortalidade e morbidade, mas também pela freqüência com que o mesmo ocorre. As maiores prevalências de baixo peso ao nascer são observadas nos países em desenvolvimento, como uma conseqüência das piores condições de vida existentes nestes locais.

$\mathrm{O}$ baixo peso ao nascer pode ser devido à menor duração da gestação, ao retardo de crescimento intra-uterino, ou ainda a uma combinação de ambos (Kramer, 1987). Villar \& Belizan (1982) apontam o retardo de crescimento intra-uterino como o maior responsável pelo baixo peso ao nascer nos países em desenvolvimento, ao passo que nos países desenvolvidos esta condição é decorrente principalmente de nascimento pré-termo.

O presente estudo teve como objetivo comparar a ocorrência de baixo peso ao nascer, assim como de nascimentos pré-termo e retardo de crescimento intra-uterino, em duas coortes de nascimento de base populacional na cidade de Pelotas, RS, nos anos de 1982 e 1993.

Tabela 1

Distribuição do peso ao nascer para os nascidos vivos. Pelotas, 1982 e 1993.

\begin{tabular}{lrrrr}
\hline Peso ao nascer $(g)$ & \multicolumn{2}{c}{1982} & \multicolumn{2}{c}{1993} \\
& $n$ & $\%$ & $n$ & $\%$ \\
\hline 1.000 & 22 & 0,4 & 18 & 0,3 \\
$1.000-1.499$ & 43 & 0,7 & 30 & 0,6 \\
$1.500-1.999$ & 109 & 1,8 & 88 & 1,7 \\
$2.000-2.499$ & 361 & 6,1 & 376 & 7,2 \\
$2.500-2.999$ & 1.392 & 23,6 & 1.310 & 25,0 \\
$3.000-3.499$ & 2.219 & 37,6 & 2.049 & 39,2 \\
$3.500-3.999$ & 1.418 & 24,0 & 1.080 & 20,6 \\
$\geq 4.000$ & 345 & 5,8 & 280 & 5,4 \\
Não pesados & 5 & & 18 & \\
& 5.914 & 100,0 & 5.249 & 100,0
\end{tabular}

\section{Metodologia}

Durante o ano de 1993, as cinco maternidades da cidade de Pelotas foram visitadas diariamente e as parturientes entrevistadas, processo semelhante ao utilizado em 1982, o que garantiu a comparabilidade dos dados. A metodologia destes estudos encontra-se descrita em outras publicações (Victora et al., 1990; Barros et al., 1990; Victora et al., 1996).

Em ambos os estudos, os entrevistadores eram estudantes de Medicina, previamente treinados. Cerca de $5 \%$ das entrevistas foram repetidas pelos supervisores para garantir a qualidade do trabalho de campo. Utilizou-se um questionário padronizado com perguntas sobre condição sócio-econômica, demográfica e utilização do programa de pré-natal, entre outras variáveis. No que diz respeito ao peso de nascimento, idade gestacional e crescimento intra-uterino, os nascimentos foram classificados da seguinte maneira:

Baixo peso ao nascer: nascidos vivos com peso inferior a $2.500 \mathrm{~g}$. O peso de nascimento foi obtido na maternidade, em balanças calibradas semanalmente pela equipe de pesquisa.

Nascimento pré-termo: nascidos vivos que apresentavam uma idade gestacional abaixo de 37 semanas, calculada a partir da data da última menstruação. Aquelas crianças que apresentavam um peso de nascimento incompatível com os padrões normais para a idade gestacional (Lubchenco et al., 1972) tiveram a sua idade gestacional considerada como desconhecida.

Retardo de crescimento intra-uterino: crianças com peso de nascimento inferior ao percentil 10 para a sua idade gestacional e sexo, de acordo com a população padrão da curva proposta por Williams (Williams et al., 1982) .

Na análise estatística utilizou-se o programa SPSS/PC+ (Norussis, 1990). Para as comparações entre proporções, utilizou-se o teste do Qui-Quadrado (Kirkwood, 1988).

\section{Resultados}

Foram identificados 6.011 e 5.304 nascimentos correspondentes a crianças cujas famílias residiam na área urbana do Município em 1982 e 1993, respectivamente. Considerando apenas os nascidos vivos, a população estudada foi de 5.914 em 1982 e 5.249 em 1993.

O baixo peso ao nascer aumentou discretamente, de 9,0\% em 1982 para 9,8\% em 1993, mas esta diferença não foi estatisticamente significativa $(\mathrm{p}=0,2)$. A incidência de nascimento pré-termo aumentou cerca de $33 \%$, de $5,6 \%$ em 
1982 para 7,5\% em $1993(\mathrm{p}<0,01)$. O retardo de crescimento intra-uterino também apresentou um aumento, embora de menor intensidade, de 15,0\% em 1982 para 17,5\% em 1993 (p<0,05).

A Tabela 1 mostra a distribuição do peso de nascimento por grupos de $500 \mathrm{~g}$, como é de praxe estudar-se na literatura internacional. Observa-se que o aumento na prevalência de baixo peso ao nascer em 1993 deveu-se à maior proporção de crianças no grupo de peso entre $2.000 \mathrm{~g}$ e $2.499 \mathrm{~g}$, que aumentou de $6,1 \% \mathrm{em}$ 1982 para $7,2 \%$ em 1993. Ao se controlar para os possíveis efeitos de confusão de situação sócio-econômica e estado nutricional materno, por meio de regressão logística, houve um importante aumento no risco de baixo peso ao nascer em 1993, da ordem de $33 \%(\mathrm{p}<0,01)$.

A Tabela 2 apresenta a prevalência de baixo peso ao nascer em 1982 e 1993, de acordo com a renda familiar em salários mínimos. Observa-se que, em ambos os períodos, há marcadas diferenças entre os grupos de renda. Em 1982, a proporção de crianças com baixo peso ao nascer foi quase três vezes maior entre as famílias de menor renda (inferior ou igual a um salário mínimo) em comparação com as de melhor situação econômica (renda acima de dez salários mínimos). As diferenças entre os grupos de renda permaneceram marcadas em 1993, com as crianças mais pobres apresentando um risco 2,4 vezes maior de nascerem com baixo peso do que as de famílias mais ricas. Cabe também ressaltar, na comparação deste indicador, que nos grupos extremos de renda - os mais pobres e os mais ricos - a prevalência de baixo peso foi menor em 1993, mas para os três grupos de renda intermediária, que são os mais numerosos, a prevalência foi maior em 1993.

O retardo de crescimento intra-uterino também apresentou uma relação inversamente proporcional com a renda familiar. Em 1982, estas diferenças foram bem marcadas e a proporção de crianças com retardo de crescimento intra-uterino foi cerca de 2,5 vezes maior entre as famílias mais pobres em comparação com as de melhor situação sócio-econômica. Em 1993, estas diferenças permaneceram bem marcadas e as crianças mais pobres apresentaram um risco 1,7 vezes maior de nascer com retardo de crescimento intra-uterino do que as de famílias ricas (Tabela 3).

Os nascimentos pré-termo não estiveram associados com a renda familiar, apesar da maior incidência observada nas famílias de baixa renda (Tabela 4 ).

A Figura 1 mostra a distribuição das crianças nascidas em 1982 e 1993 conforme o peso ao nascer e idade gestacional. O aumento no

\begin{tabular}{|c|c|c|}
\hline \multicolumn{3}{|c|}{$\begin{array}{l}\text { Prevalência de baixo peso ao nascer de acordo com a renda familiar. } \\
\text { Pelotas, } 1982 \text { e } 1993 .\end{array}$} \\
\hline $\begin{array}{l}\text { Renda familiar } \\
\text { em salários mínimos }\end{array}$ & $\begin{array}{l}1982^{*} \\
\%\end{array}$ & $\begin{array}{l}1993^{*} \\
\%\end{array}$ \\
\hline$\leq 1$ & 15,0 & 11,1 \\
\hline $1,1-3$ & 7,8 & 10,5 \\
\hline $3,1-6$ & 7,2 & 9,6 \\
\hline $6,1-10$ & 6,6 & 7,7 \\
\hline$>10$ & 5,4 & 4,7 \\
\hline \multirow[t]{2}{*}{ Total } & 9,0 & 9,8 \\
\hline & $(5.914)$ & $(5.249)$ \\
\hline
\end{tabular}

* $p<0,05$

Tabela 3

Prevalência de retardo de crescimento intra-uterino de acordo com a renda familiar. Pelotas, 1982 e 1993.

\begin{tabular}{lcc}
\hline $\begin{array}{l}\text { Renda familiar } \\
\text { em salários mínimos }\end{array}$ & $\begin{array}{c}1982^{\star} \\
\%\end{array}$ & $\begin{array}{c}1993^{*} \\
\%\end{array}$ \\
\hline$\leq 1$ & 21,8 & 21,9 \\
$1,1-3$ & 15,3 & 18,7 \\
$3,1-6$ & 12,7 & 15,9 \\
$6,1-10$ & 6,2 & 11,9 \\
$>10$ & 8,8 & 12,3 \\
& & 17,5 \\
Total & 15,0 & $(5.249)$ \\
\hline
\end{tabular}

* $p<0,05$

Tabela 4

Incidência de nascimento pré-termo de acordo com a renda familiar. Pelotas, 1982 e 1993.

\begin{tabular}{lcc}
\hline $\begin{array}{l}\text { Renda familiar } \\
\text { em salários mínimos }\end{array}$ & $\begin{array}{c}1982 \\
\%\end{array}$ & $\begin{array}{c}1993 \\
\%\end{array}$ \\
\hline$\leq 1$ & 7,2 & 8,6 \\
$1,1-3$ & 5,1 & 8,3 \\
$3,1-6$ & 5,0 & 6,5 \\
$6,1-10$ & 6,5 & 5,4 \\
$>10$ & 4,7 & 6,2 \\
& & 7,5 \\
Total & 5,6 & $(5.249)$ \\
\hline
\end{tabular}


Distribuição da população estudada de acordo com o baixo peso ao nascer, retardo de crescimento intra-uterino e nascimento pré-termo. Pelotas, 1982 e 1993.

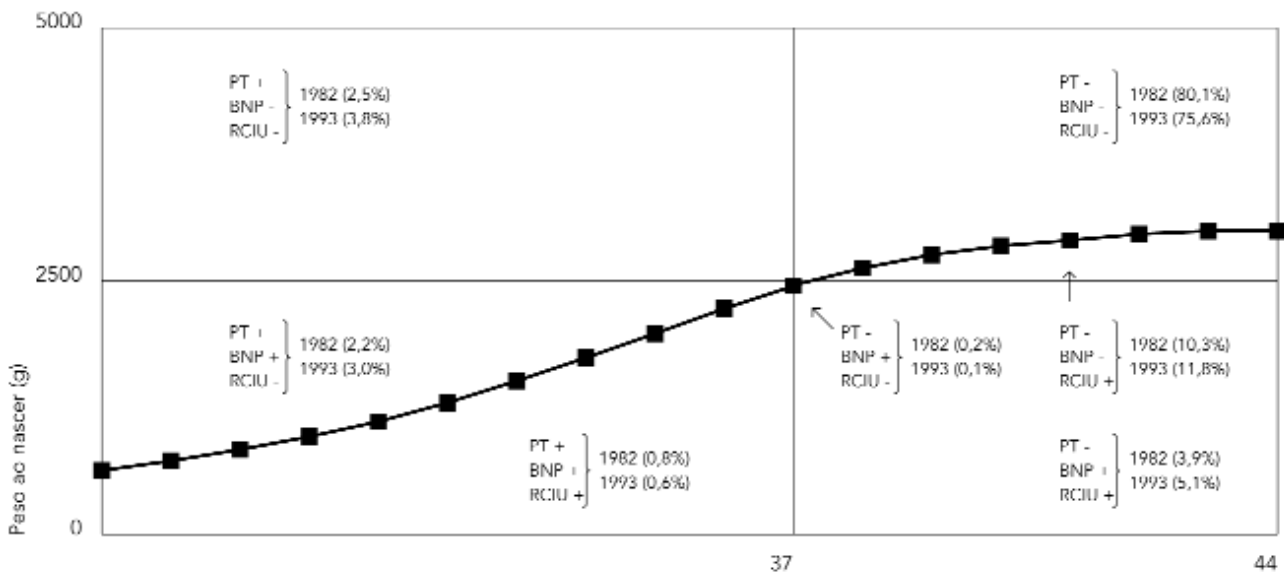

PI - nascimento prè-termo BNP - baixo peso ao nascer RCIU - retardo de crescimento intra uterino baixo peso ao nascer, em 1993, foi decorrente tanto da maior proporção de nascimentos de crianças pré-termo com peso adequado para a idade gestacional, como de crianças nascidas a termo com retardo de crescimento intra-uterino. No que diz respeito ao nascimento pré-termo, observou-se uma redução de $50 \%$ na proporção do mesmo entre as crianças com peso acima de $2.500 \mathrm{~g}$.

\section{Discussão}

Estes dois estudos perinatais de base populacional, realizados em Pelotas em 1982 e 1993, cobriram mais de $99 \%$ dos nascimentos hospitalares ocorridos na cidade nestes anos, constituindo-se em uma base de dados que raramente é disponível, mesmo em países desenvolvidos. Nesta cidade, os nascimentos domiciliares são muito raros (Victora et al., 1990), sendo mínima, portanto, a possibilidade de viés de seleção. Estes estudos permitiram a comparação da evolução da saúde perinatal em um período de 11 anos.

Houve uma evidente melhora nas condições sócio-econômicas e nutricionais das mães, assim como um aumento na cobertura pré-natal, conforme descrito em outro artigo deste suplemento (Costa et al., 1996). Apesar destas melhoras, houve um aumento na prevalência de baixo peso ao nascer, de 9,0\% em 1982 para 9,8\% em 1993, diferença que não foi estatistica- mente significativa na análise bivariada. Entretanto, quando os possíveis efeitos de confusão de situação sócio-econômica e estado nutricional materno foram controlados na análise multivariada, mediante regressão logística, houve um importante aumento no risco de baixo peso ao nascer em 1993, da ordem de 33\% ( $<<0,01)$.

Nos Estados Unidos, também tem sido observado um aumento na prevalência de baixo peso ao nascer, que foi de $6,8 \%$ em 1985 e $7,1 \%$ em 1992 (Wegman, 1987; Wegman, 1993). Amini e colaboradores (Amini et al., 1994) também observaram, no período de 1975 a 1992, em Cleveland, um aumento na prevalência de baixo peso ao nascer, de $12,7 \%$ para $17,3 \%$, e na incidência de nascimento pré-termo, de 3,3\% para 7,8\%.

Portanto, é necessário entender, através de análises complementares, por que, em Pelotas, no período de 1982 e 1993, apesar de melhorias na situação nutricional e socio-econômica maternas e na cobertura de saúde, houve maior número de baixo peso ao nascer, ao contrário do que seria esperado. Este crescimento foi decorrente tanto do aumento na proporção de nascimentos pré-termo, como de retardo de crescimento intra-uterino. Nos Estados Unidos, o maior número de cesarianas foi considerado como um dos principais responsáveis por este aumento. Esta probabilidade já foi alvo de uma análise preliminar em nosso banco de dados, mas as cesarianas parecem não ter sido responsáveis pelo aumento do baixo peso ao nascer em nosso meio. 


\section{Referências}

AMINI, S. B.; DIERKER, L. R. J.; CATALANO, P. M.; ASHMEAD, G. G. \& MANN L. I., 1994. Trends in an obstetric patient population: An eighteen-year study. American Journal of Obstetrics and Gynecology, 171:1014-21.

BARROS, F. C.; VICTORA, C. G. \& VAUGHAN, J. P., 1990. The Pelotas birth cohort study, 1982-1987. Strategies for following up 6,000 children in a developing country. Perinatal and Pediatric Epidemiology, 4:267-82.

COSTA, J. S. D.; VICTORA, C. G.; BARROS, F. C.; HALPERN, R.; HORTA, B. L. \& MANZOLLI P., 1996. Assistência médica em duas coortes de base populacional no Sul do Brasil: tendências e diferenciais. Cadernos de Saúde Pública, 12(supl. 1):59-66.

KIRKWOOD, B. R., 1988. Essentials of Medical Statistics. Oxford: Blackwell Scientific Publications.

KRAMER, M. S., 1987. Determinants of low birth weight: methodological assessment and metaanalysis. Bulletin of the World Health Organization, 65:665-737.

LUBCHENCO, L. O.; SEARLS, D. T. \& BRAZIE, J. V., 1972. Neonatal mortality rate: Relationship to birth weight and gestational age. Journal of Pediatrics, 81:814-22.

MCCORMICK, M. C., 1985. The contribution of low birth weight to infant mortality and childhood morbidity. New England Journal of Medicine, 312:82-90.
NORUSSIS, M. J.,1990. SPSS/PC+: Statistical Package for Social Science. Chicago: SPSS Inc.

VICTORA, C. G.; BARROS, F. C. \& VAUGHAN, J. P., 1989. Epidemiologia da Desigualdade: Um Estudo Longitudinal de 6.000 Crianças Brasileiras. São Paulo: Cebes/Hucitec.

VICTORA, C. G.; BARROS, F. C.; HALPERN, R.; MENEZES, A. M. B.; HORTA, B. L.; TOMASI, E.; WEIDERPASS, E.; CESAR, J. A.; OLINTO, M. T.; GUIMARÃES, P. R. V.; GARCIA, M. M. \& VAUGHAN, J. P., 1996. Estudo longitudinal da população materno-infantil de Pelotas, RS, 1993: aspectos metodológicos e resultados preliminares. Revista de Saúde Pública, 30:34-45.

VILLAR, J. \& BELIZAN, J. M., 1982. The timing factor in the pathophysiology of the Intrauterine Growth Retardation Syndrome. Obstetrical and Gynecological Survey, 37:499-506.

WILLIAMS, R. L.; CREASY, R. K.; CUNNINGHAM, G. C.; HAWES, W. E.; NORRIS, F. D. \& TASHIRO, M., 1982. Fetal growth and perinatal viability in California. Obstetrics \& Gynecology, 59:624-32.

WEGMAN, M. E., 1987. Annual summary of vital statistics-1986. Pediatrics, 80:817-27.

WEGMAN, M. E., 1993. Annual summary of vital statistics-1992. Pediatrics, 92:743-54. 